\title{
SOME OPERATOR MONOTONE FUNCTIONS ${ }^{1}$
}

\author{
GERT K. PEDERSEN
}

ABSTRACT. A short proof is given based on $C^{*}$-algebra theory for the well-known theorem that if $S$ and $T$ are bounded selfadjoint operators on a Hilbert space such that $0 \leqq S \leqq T$ then $S^{\alpha} \leqq T^{\alpha}$ for each $0 \leqq \alpha \leqq 1$.

THEOREM. If $S$ and $T$ are bounded selfadjoint operators on a Hilbert space $\mathfrak{H}$ such that $0 \leqq S \leqq T$ then $S^{\alpha} \leqq T^{\alpha}$ for each $\alpha$ in the interval $[0,1]$.

REMARK. The theorem says that each function $t \rightarrow t^{\alpha}$, with $0 \leqq \alpha \leqq 1$, is operator monotone on the set of positive operators in $B(\mathfrak{H})$. This was first proved by $\mathrm{K}$. Löwner, who gave a complete description of operator monotone functions. Later T. Ogasawara gave a short proof of the operator monotonicity for the square root function. We present here a simple proof based on $C^{*}$-algebra theory.

PRoof. If $0 \leqq S \leqq T$ then $S+\varepsilon I \leqq T+\varepsilon I$ for each $\varepsilon>0$; and $S+\varepsilon I$ and $T+\varepsilon I$ are both invertible. Since $(S+\varepsilon I)^{\alpha}$ converges to $S^{\alpha}$ in norm when $\varepsilon \rightarrow 0$ for each $\alpha>0$, and since the positive operators in $B(\mathfrak{H})$ form a norm closed set, it suffices to prove the theorem assuming that $S$ and $T$ are invertible. (The case $\alpha=0$ can be verified directly, since $S^{0}$ is the range projection of $S$.)

Let $E$ denote the set of exponents $\alpha$ in $[0,1]$ for which the function $t \rightarrow t^{\alpha}$ is operator monotone. Trivially $0 \in E$ and $1 \in E$. Since the function $\alpha \rightarrow S^{\alpha}$ is continuous from $[0,1]$ to $B(\mathfrak{H})$ in the norm topology we see that $E$ is a closed set. The proof will be complete when we show that $E$ is convex.

Take $\alpha$ and $\beta$ in $E$. Then $S^{\alpha} \leqq T^{\alpha}$; hence $T^{-\alpha / 2} S^{\alpha} T^{-\alpha / 2} \leqq I$. It follows that $\left\|S^{\alpha / 2} T^{-\alpha / 2}\right\| \leqq 1$. Similarly $\left\|S^{\beta / 2} T^{-\beta / 2}\right\| \leqq 1$. With $\rho(A)$ the spectral radius of an operator $A$ we have $\rho(A B)=\rho(B A)$. Therefore

$$
\begin{aligned}
\rho\left(T^{-(\alpha+\beta) / 4} S^{(\alpha+\beta) / 2} T^{-(\alpha+\beta) / 4}\right) & =\rho\left(T^{(\alpha-\beta) / 4} T^{-(\alpha+\beta) / 4} S^{(\alpha+\beta) / 2} T^{-(\alpha+\beta) / 4} T^{-(\alpha-\beta) / 4}\right) \\
& =\rho\left(T^{-\beta / 2} S^{(\alpha+\beta) / 2} T^{-\alpha / 2}\right) \leqq\left\|T^{-\beta / 2} S^{(\alpha+\beta) / 2} T^{-\alpha / 2}\right\| \\
& \leqq\left\|T^{-\beta / 2} S^{\beta / 2}\right\|\left\|S^{\alpha / 2} T^{-\alpha / 2}\right\| \leqq 1
\end{aligned}
$$

Received by the editors April 27, 1972.

AMS 1970 subject classifications. Primary 47B15; Secondary 46L05.

Key words and phrases. Operator monotone functions, $C^{*}$-algebras, positive operators.

1 The preparation of this paper was supported in part by NSF Grant 28976X.

(c) American Mathematical Society 1972 
It follows that $T^{-(\alpha+\beta) / 4} S^{(\alpha+\beta) / 2} T^{-(\alpha+\beta) / 4} \leqq I$, so that $S^{(\alpha+\beta) / 2} \leqq T^{(\alpha+\beta) / 2}$. This shows that $(\alpha+\beta) / 2 \in E$ which completes the proof.

\section{REFERENCES}

1. J. Dixmier, Les $C^{*}$-algèbres et leurs représentations, Cahiers Scientifiques, fasc. 29, Gauthier-Villars, Paris, 1964. MR 30 \#1404.

2. K. Löwner, Über monotone matrixfunctionen, Math. Z. 38 (1934), 177-216.

3. T. Ogasawara, $A$ theorem on operator algebras, J. Sci. Hiroshima Univ. Ser. A 18 (1955), 307-309. MR 17, 514.

Department of Mathematics, University of Copenhagen, Copenhagen, Denmark

Current address: Matematisk Institut, Universitetsparken 5, 2100 Copenhagen, Denmark 